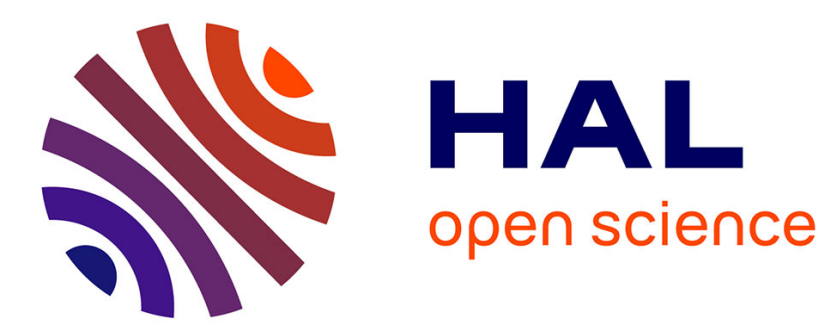

\title{
Time fractional derivative for frequency effect in ferroelectrics
}

Benjamin Ducharne, G. Sebald, D. Guyomar

\section{To cite this version:}

Benjamin Ducharne, G. Sebald, D. Guyomar. Time fractional derivative for frequency effect in ferroelectrics. 2009 18th IEEE International Symposium on the Applications of Ferroelectrics (ISAF), Aug 2009, Xian, China. 10.1109/ISAF.2009.5307619 . hal-02560951

\section{HAL Id: hal-02560951 \\ https://hal.science/hal-02560951}

Submitted on 2 May 2020

HAL is a multi-disciplinary open access archive for the deposit and dissemination of scientific research documents, whether they are published or not. The documents may come from teaching and research institutions in France or abroad, or from public or private research centers.
L'archive ouverte pluridisciplinaire HAL, est destinée au dépôt et à la diffusion de documents scientifiques de niveau recherche, publiés ou non, émanant des établissements d'enseignement et de recherche français ou étrangers, des laboratoires publics ou privés. 


\title{
Time fractional derivative for frequency effect in ferroelectrics
}

\author{
B. Ducharne, G. Sebald, D. Guyomar \\ Laboratoire de Génie Electrique et Ferroélectricité - INSA de Lyon \\ Bât. Gustave FERRIE, 8 rue de la physique, 69621 Villeurbanne cedex, FRANCE
}

\begin{abstract}
The present article proposes a dynamical model to obtain ferroelectric hysteresis dynamics based on fractional derivatives (Polarization versus Electric field curves). The consideration of a fractional derivative term widely increases the frequency bandwidth of the accuracy of the traditional hysteresis models. For PZT bulk ceramics, the order of the fractional derivative has been found to be 0.5 using experimental data as $10^{-3} \mathrm{~Hz}<f<100 \mathrm{~Hz}$. For these excitation frequency levels, simulation tests provided good results regarding the comparison of the fractional model and experimental results. With the same set of parameters, it is possible to take into account the nonlinear behavior as $f \rightarrow 0$ : creep phenomenon, ageing ... Next, the model was tested on large frequency bandwidths ( $>6$ decades) and validated with success using the comparison between simulation tests and the only experimental results available in literature obtained in such conditions by Liu and al (J. Phys.:Condens. Matter., 2004, vol.16, pp.1189-1195) for BNT thin film samples. At such frequency levels $\left(>10^{2} \mathrm{~Hz}\right)$, due to power limitations, no ceramic bulk's experimental results were available.
\end{abstract}

\section{INTRODUCTION}

Ferroelectric materials are widely applied as actuators and sensors, as they exploit the intrinsic coupling between electro-mechanical, electro-optical, and thermoelectrical properties [1]. When loaded with an electric field or a mechanical stress, ferroelectrics display timedependent strain and electric displacement behavior. Understanding this behavior is essential for the design of industrial applications. Nonlinear polarization leads to irreversible changes in the ultimate electromechanical properties, which, in turn, induce mismatches between transducers and their controlling system. In recent years, significant progress has been made with regard to modeling the material response of ferroelectrics and obtaining a comprehension of the underlying phenomena. Rate-independent approaches, such as Preisach models [2], micromechanical models [3], and phenomenological models, have been used to describe the ferroelectric hysteresis under a constant loading or over a limited frequency bandwidth [4]. However, a model that is accurate over a large frequency range ( $>10$ decades) is still missing. The polarization response is dependent on the excitation frequency, $f$. Nevertheless, the amplitude, $\mathrm{E}_{0}$, and the origin of excitation (electric field, mechanical stress, thermal variations, etc.) are also major parameters. Polarization phenomena represent complex and dynamic processes including the nucleation of domains, the forward and sideways growth of interacting domains, as well as domain coalescence. It is particularly interesting to study the response of the hysteresis when varying the frequency and the excitation amplitude. The hysteresis area, $\angle \mathrm{A}\rangle$, is related to the energy dissipation in one reversal cycle. Also, the remnant polarization, $\mathrm{Pr}$, and the coercive field, Ec, which are determined from hysteresis loop, are two major parameters for advanced ferroelectric devices and depend strongly on the excitation frequency and amplitude. Consequently, the understanding and modeling of dynamic polarization behaviors could provide precious tools for the computation of dissipation losses and dielectric responses of ferroelectric devices (such as piezoelectric actuators).

The present paper proposes a formulation based on non-entire derivatives for the modeling of the dynamic hysteresis over a large frequency bandwidth. As $f \rightarrow 0$, the model has been validated by carrying out a comparison between simulation and experimental data of mechanical creep. As $f \rightarrow \infty$, the model was tested and validated using the comparison between simulation tests and the only experimental results available in literature obtained in such conditions by Liu and al for BNT thin film samples [5].

The present paper first exposes the configuration of the two majors terms of the model: a quasi-static hysteresis contribution, with the form of a damping force (a negative first-order polarization time derivative corresponding to the poling current); and a timedependent loss term, corresponding to the product of a material constant, $\rho$, and a fractional polarization derivative term, $\mathrm{d}^{\alpha} \mathrm{P} / \mathrm{dt}^{\alpha}(\alpha € \mathrm{R})$. Then the wide frequency bandwidth accuracy of the model is illustrated by testing its accuracy; when $f \rightarrow 0$, and when $f \rightarrow \infty$.

\section{QUASI-STATIC MODEL}

A static contribution signifies observing a loop-like hysteresis when plotting the spontaneous polarization, $\mathrm{P}$, against $\mathrm{E}$ for very low frequencies $(f<<1 \mathrm{~Hz})$. At such frequency levels, wall movements are assumed to undergo a mechanical-like dry friction. A static equation based on its mechanical dry-friction counterpart has been established in order to account for this property. The generation of a major $\mathrm{P}(\mathrm{E})$ hysteresis loop was observed with a good approximation by the translation of an anhysteretic curve; the loop also depended on the sign of the time derivative of the polarization. This translation is equal to the coercive field, Ec. A correct description of the major hysteresis loops observed during steady state of the ceramic under a high-amplitude electric field $\left(\mathrm{E}_{0}>>\mathrm{Ec}\right)$ can be obtained with the equation related to a 
single domain wall model. But as illustrated in [6], the polarization remains null until a value of the electric field becomes equal to the coercive field. This signifies that the first polarization curve cannot be correctly reproduced by only one equation, which is true for all simulated minor loops. A good global ferroelectric material model needs to take into account the set of similar behaviors of each domain wall and is characterized by its own coercive field. More realistic cycles are obtained by introducing a distribution of a basic element (spectrum), characterized by its own coercive fields in addition to its own weight:

$$
\begin{gathered}
\text { if }: \quad E(t)>f^{-1}\left(P_{i}(t)\right)+E c_{i} \\
P_{i}(t+d t)=f\left(E(t+d t)-E c_{i}\right) \\
\text { if }: \quad E(t)<f^{-1}\left(P(t)_{i}\right)-E c_{i} \\
P_{i}(t+d t)=f\left(E(t+d t)+E c_{i}\right) \\
\text { if }: f^{-1}\left(P(t)_{i}\right)-E c_{i}<E(t)<f^{-1}\left(P(t)_{i}\right)+E c_{i} \\
P_{i}(t+d t)=P_{i}(t) \\
\sum_{i=1}^{k} \operatorname{Spectrum}(i) . P_{i}=P
\end{gathered}
$$

(1)

Here, $f(\mathrm{E})$ (reciprocally $f^{-1}(\mathrm{P})$ ) represents the behavior of a non-linear dielectric (without hysteresis), and together with the parameters $(\gamma, \sigma)$, the function can be obtained by fitting these parameters to the anhysteretic curve of a perfect dielectric given function, for example:

$$
\begin{aligned}
f(E)=\sigma \cdot \tan ^{-1}\left(\frac{E}{\gamma}\right) \\
f^{-1}(P)=\gamma \cdot \tan \left(\frac{P}{\sigma}\right)
\end{aligned}
$$

(2)

Spectrum(i) represents the distribution of elementary loops [6].
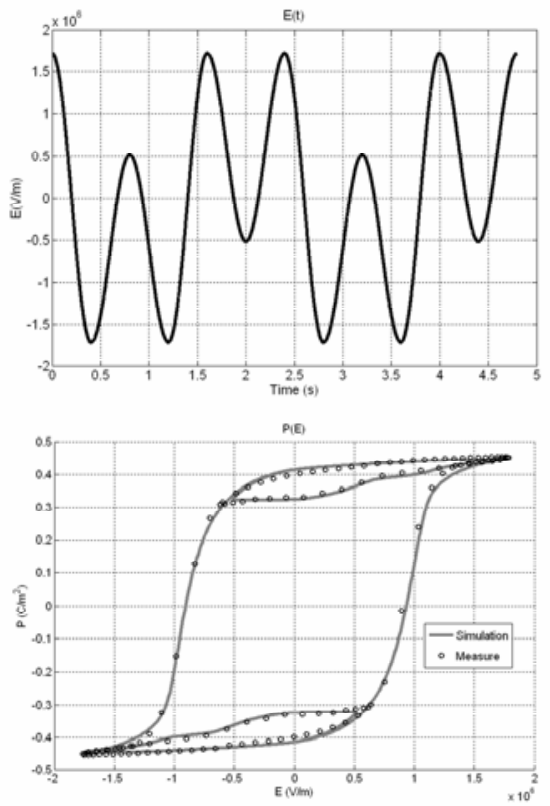

Fig. 1. Comparison simulation (grey)/measure (black) for unsymmetrical static excitation.

\section{DYNAMICAL MODEL}

The dynamical effect is usually represented by adding the product of a resistive term $\rho$ and the derivative polarization to the static contribution $(\alpha=0$ in equations $\left.n^{\circ} 3\right)[6]:$

$$
\begin{aligned}
& \text { if }: E(t)>f^{-1}\left(P_{i}(t)\right)+E c_{i}+\rho \cdot \frac{d^{\alpha} P_{i}(t)}{d t^{\alpha}} \\
& P_{i}(t+d t)>f\left(E(t+d t)-E c_{i}-\rho \cdot \frac{d^{\alpha} P_{i}(t)}{d t^{\alpha}}\right) \\
& \text { if }: E(t)>f^{-1}\left(P_{i}(t)\right)-E c_{i}-\rho \cdot \frac{d^{\alpha} P_{i}(t)}{d t^{\alpha}} \\
& P_{i}(t+d t)>f\left(E(t+d t)+E c_{i}+\rho \cdot \frac{d^{\alpha} P_{i}(t)}{d t^{\alpha}}\right) \\
& \text { if : } f^{-1}\left(P_{i}(t)\right)-E c_{i}-\rho \cdot \frac{d^{\alpha} P_{i}(t)}{d t^{\alpha}}<E(t)<f^{-1}\left(P_{i}(t)\right)+E c_{i}+\rho \cdot \frac{d^{\alpha} P_{i}(t)}{d t^{\alpha}} \\
& P_{i}(t+d t)=P_{i}(t) \\
& \sum_{i=1}^{k} \operatorname{Spectrum}(i) \cdot P_{i}=P
\end{aligned}
$$

Experimental data shows that this dynamical modelling is accurate for a restrained frequency bandwidth [7]. The dynamical contribution $\rho . d P / d t$ leads to an overestimation of the high frequency component, as illustrated in the loop area versus frequency curve (figure $\mathrm{n}^{\circ} 4$ ) obtained for a given $\mathrm{E}_{0}$. This overestimation is corrected by defining an operator that balances the low and high frequency components in a different way than a linear time derivative. Such operators exist in the framework of fractional calculus: the so-called non-entire or fractional derivatives. Fractional derivatives involve raising derivatives to non-integer orders. This means that the order of the derivative can be either a real or complex number. In general, the fractional derivative of a function $f(t)$ is the convolution of the $f(t)$ function and $t^{n} H(t) / \Gamma(1-$ $\mathrm{n})$, where $\Gamma(\mathrm{n})$ is the gamma function and $\alpha$ the order of the fractional derivative. From a spectral point of view, this formulation means that the frequency spectrum $f(\omega)$ of $f(t)$ will be multiplied by $(j \omega)^{n}$, instead of $j \omega$, for first order derivatives. Other definitions of fractionnal derivatives exist, and among these, the Grunwald derivative is well suited for numerical implementation; it will be used in this paper.

The Grunwald derivative is:

$$
\begin{aligned}
D^{n} f(t) & =\lim _{h \rightarrow 0} \frac{1}{h^{n}} \sum_{k=0}^{\infty}(-1)^{k}\left(\begin{array}{l}
n \\
k
\end{array}\right) f(t-k h) \\
\left(\begin{array}{l}
n \\
k
\end{array}\right) & =\frac{n(n-1)(n-2) \ldots(n-k+1)}{k !}
\end{aligned}
$$

Here, $\mathrm{h}$ is the sampling time period, and $\mathrm{n}$ is the order of the fractional derivative. 
Dynamical unsymmetrical hysteresis loops are obtained by introducing the fractional operator into system $\mathrm{n}^{\circ} 3(0<\alpha<1)$.

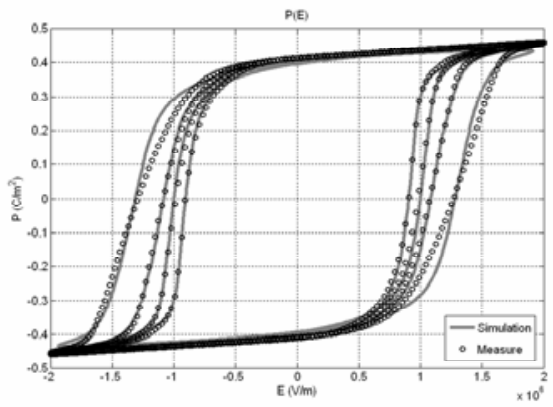

Fig. 2. Comparison simulation (grey)/measure (black) for dynamical high amplitude excitation $\left(\mathrm{f}=1,10,10^{2}, 10^{3} \mathrm{~Hz}\right)$.

\section{EXPERIMENTAL PROCEDURE}

A typical industrial soft PZT composition (P188 obtained from Quartz \& Silice, France, Navy type II) was the object of the present study, and the main characteristics of the material are given in Table 1 .

\begin{tabular}{|l|c|c|c|}
\hline \multicolumn{1}{|c|}{ Parameters } & Symbol & Units & Typical values \\
\hline Density & $\rho$ & $10^{3} \mathrm{~kg} \cdot \mathrm{m}^{-3}$ & 7.7 \\
Poisson's constant & $\sigma$ & & 0.3 \\
Curie point & $\mathrm{TC}$ & ${ }^{\circ} \mathrm{C}$ & 340 \\
Dielectric permittivity & $\varepsilon^{T}{ }_{33} / \varepsilon_{0}$ & & 1850 \\
Piezoelectric & $\mathrm{d} 33$ & $\mathrm{pC} / \mathrm{N}$ & 425 \\
coefficient & \multicolumn{2}{|l}{} \\
\hline
\end{tabular}

Tab. 1. The main characteristics of a standard P188 ceramic.

Cylindrical specimens (diameter 6.35mm, height: $2 \mathrm{~mm}$ ), electroded with fire-on paste were subjected to ahigh amplitude excitation in an electric field by an Optilas Trek 10-kV high voltage supply. Constant temperature conditions and free mechanical properties of the sample were assumed. To avoid thermal drifts and dielectric breakdown, the samples were placed in an oil bath at room temperature. Electric displacements were calculated by current measurements (Keithley amplifier 5011), and the polarization fields were computed by integrating the currents. For the mechanical creep measurements, the field-induced thickness strain was measured using a double beam laser interferometer (Agilent 10889B), with a precision of $10 \mathrm{~nm}$. The sample was placed on a horizontal brass disc in order to ensure a good mechanical base. A second brass disc was placed on the upper side of the film, permitting the application of a bipolar electric field.

\section{EXPERIMENTAL RESULTS}

\section{Dynamic result as $f \rightarrow 0$}

For varying loading rates or when accuracy over long time periods is required, it is important not to neglect rate effects and creep in remnant strain and polarization. Creep phenomena represent major limitations in numerous applications of PZT actuators (micro-positioning, surface measurements, etc.), and can be described as a picture of the dynamical behavior for $f$ $\rightarrow 0$. By successfully modeling the mechanical creep, one can admit and validate the model accuracy for long time variations. Modeling of the creep behavior is also a challenging means of validating the proposed model. Experimental measurements on PZT ferroelectric ceramics demonstrate how the polarization falls under compressive stress. As a consequence of this decrease in polarization, degradations of the piezoelectric properties (caused by rearrangements of the ferroelectric domain) can be observed. Similarly to the electric field, mechanical stresses also induce $\Delta \mathrm{P}$ variations; however, such $\Delta \mathrm{P}$ variations demonstrate an opposite polarization sign. A mechanical stress, $\mathrm{T}$, can be introduced in the model as an equivalent to the electric field ( $\mathrm{F}=$ applied force $=\mathrm{E}=\mathrm{h}(\mathrm{P}) . \mathrm{T})$. Here, $\mathrm{h}(\mathrm{P})$ is a specific function permitting a modification of the symmetry described above. Moreover, $h(P)$ must be an odd function: $h(P)=\lambda$ $\mathrm{P}$ can be used, where the value of $\lambda$ is a fit based on experimental measurements. Then the following equation linking strain $\mathrm{S}$ and polarization $\mathrm{P}$ is used.

$$
S=-\lambda \int_{0}^{P(E)} P \frac{d P}{d E} d E=-\lambda \int_{0}^{P(E)} P d P
$$

Eq. (5), describing the relation between the mechanical strain and the electrical excitation, has been established and validated in [9]. It is valid for large and straight excitation amplitudes. By assuming the reversibility of the converse and direct piezoelectric effect $(\partial \mathrm{P} / \partial \mathrm{T}=\partial \mathrm{S} / \partial \mathrm{E})$, and by further substituting the partial derivative $\partial \mathrm{P} / \partial \mathrm{T}$ by $-\lambda \mathrm{P} \partial \mathrm{P} / \partial \mathrm{E}$, the strain expression can be given as:

$$
-\lambda P \cdot \frac{\partial P}{\partial T}=\frac{\partial P}{\partial E} \rightarrow S=-\lambda\left[\frac{p^{2}}{2}\right]_{0}^{P(E)}=-\lambda\left[P^{2}(E)-P^{2}(0)\right]
$$

The strain can finally be obtained as a function of $\mathrm{P}^{2}$, and a combination of previous equation gives the strain creep in simulation. Here, the shape of the creep answer is directly dependent on the non-entire derivative coefficient $\alpha$, as illustrated in Figure 3. As for $\S 1$ the best results are obtained by fixing $\alpha$ to 0.5 .

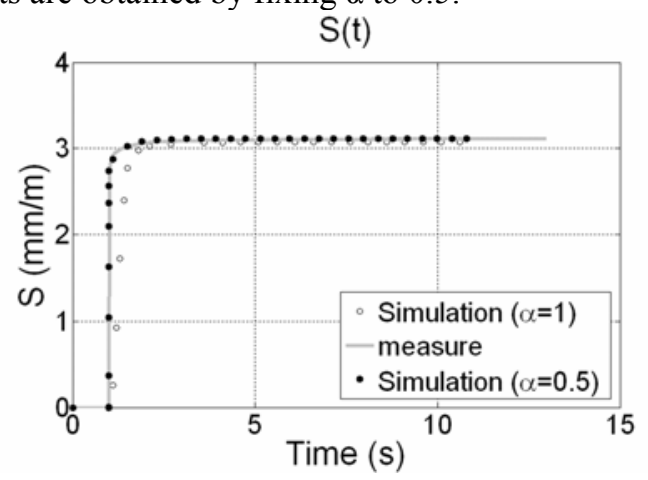

Fig. 3: The comparison between measured $(\alpha=5)$ and 
simulated strain data for long times (mechanical creep).

\section{Dynamic result as $f \rightarrow \infty$}

As a final validation of the model, a comparison has been done between the experimental results obtained by Liu and al. [10] and the fractional model for large electric field dielectric behavior (with $\mathrm{E}>\mathrm{Ec}$ ). These experimental results were obtained for a large frequency bandwidth (1 to $10^{6} \mathrm{~Hz}$ ) for $\mathrm{Nd}$-substituted $\mathrm{Bi}_{4} \mathrm{Ti}_{3} \mathrm{O}_{12}$ thin films. The electrical field $\mathrm{E}_{0}$ range is $100-400 \mathrm{kV} / \mathrm{cm}$. The characterization of the samples has been done using Sawyer-Tower circuit. As the shapes of the hysteresis loops and the physical behaviors are different to the ceramic sample P188 previously used to define the model, a new set of parameters is necessary. In the case of $\mathrm{Bi}_{3.15} \mathrm{Nd}_{0.85} \mathrm{Ti}_{3} \mathrm{O}_{12}$, Liu and al thin film samples, the best results were obtained with $\alpha=0.4, \rho=7.10^{5}$.

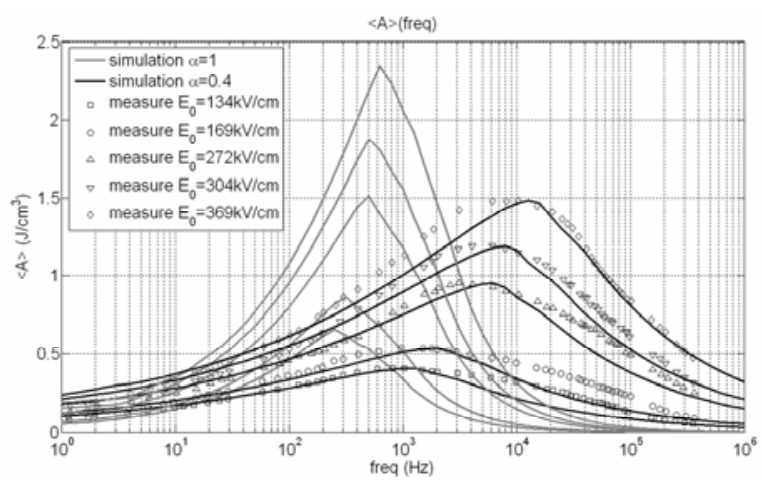

Fig. 4: Comparion simulations $(\alpha=0.5, \alpha=1)$ /measures $<\mathrm{A}>$ (freq) curves for large frequency bandwidth excitation.

As it has already been observed as $f \rightarrow 0$ and $\rightarrow \infty$, we notice good fitting between the fractional model and the measures for large frequency bandwidth type excitation. It is particularly interesting to conclude that a single set of parameters allows to perfectly reproduce the characteristics tendencies of the $\langle\mathrm{A}\rangle$ (freq) curves (increasing rates, peak position and decreasing last part ...), we prove here that only fractional operator can take into account with success all the dynamical behaviors of a ferroelectric material $\left(f \epsilon\left[10^{-3} ; 10^{6}\right] \mathrm{Hz}\right)$.

\section{CONCLUSION}

Frequency effects in ferroelectric materials may be a major limitation for high accuracy piezoactuators, Ferroelectric RAMS for example. From a more fundamental point of view, it is a challenging question: How to model losses that are obviously neither viscous nor static. Indeed, we demonstrate here via experimental on a soft PZT that static and/or viscous losses associated to a hysteretic model are completely unable to model frequency effect of ferroelectric Polarization - Electric field hysteresis curve (PE cycle).

Scaling laws were developed as a first attempt to tackle investigations on those behaviors, and frequency exponent of 0.3 up to 0.6 were found. It is an interesting way to understand losses in ferroelectrics, but this doesn't constitute a model suitable for simulating ferroelectric behavior. In this work, we aim at showing the effectiveness of a non-integer time derivation of the polarization in association to a hysteretic model (which is based on a dry friction mechanism). Such a model give an outstanding accuracy compared to experiments over 6 decades of frequencies - and probably more if we could overcome experimental constraints of power limitation. It can then be compared of course with scaling laws, but such a model is accurate also for frequencies ranges were scaling laws are totally ineffective (in the vicinity of the peaks of Fig. 4)

Time fractional derivative not only gives excellent results for PE cycles for different frequencies, is can also explain creep behavior as shown in the second part of this work.

\section{REFERENCES}

[1] H. Cao, A.G. Evans, "Nonlinear deformation of ferroelectric ceramics", J. Am. Ceram. Soc., vol.76, 890896,1993

[2] G.Robert, D. Damjanovic, and N. Setter, "Preisach modeling of piezoelectric nonlinearity in ferroelectric ceramics. J.Appl. Phys., vol.89, pp.5067-5074, 2001.

[3] J.E. Huber, N.A. Fleck, C.M. Landis and R.M. McMeeking, "A constitutive model for ferroelectric polycrystals", J. Mech. Phys. Solids, vol.44, pp.1663$1697,1999$.

[4] A.C.F. Cocks and R.M. McMeeking, "A phenomenological constitutive law for the behavior of ferroelectric ceramics", Ferroelectrics, vol.228, pp. 219228, 1999.

[5] J.M.Liu, B.Pan, H.Yu, and S.T.Zhang, "Dynamic hysteresis dispersion scaling of ferroelectric Ndsubstitued $\mathrm{Bi}_{4} \mathrm{Ti}_{3} \mathrm{O}_{12}$ thin films", J. Phys.:Cond. Matt., vol.16, pp.1189-1195, 2004.

[6] D.Guyomar, B.Ducharne, G.Sebald, "Using the fractional derivative operator for modeling dynamical polarization behaviour versus frequency and electric field amplitude", IEEE Trans. On Ultrason. Ferr. and Freq. Cont.

[7] D.Guyomar, B.Ducharne, G.Sebald, "Dynamical hysteresis model of ferroelectric ceramics under electric field using fractional derivatives", J. Phys. D: Appl. Phys., 2007.

[8] A.K. Grünwald, "Ueber begrenzte derivationen und deren Anwendung", Zeitschrift für mathematik und physik, XII(6), pp.441-480, 1867.

[9] D.Guyomar, B.Ducharne, G.Sebald, "Time fractional derivatives for voltage creep in ferroelectric materials: theory and experiment", J. Phys.D: Appl.Phys., 2008, vol.41, pp. 125410. 